\title{
Combining ability and heterosis analyses for oil and healthy fatty acid composition in groundnut (Arachis hypogaea L.)
}

\author{
Md. Rayhan Sikder ${ }^{1,2}$, Md. Abul Kalam Azad ${ }^{2}$, Sariful Haque Bhuiya ${ }^{2}$, Khondakar Sumsul Arefin², Md. \\ Mahbubul Hasan Shohag ${ }^{2} \&$ Mohammad Anwar Hossain ${ }^{1 *}$ \\ ${ }^{1}$ Department of Genetics and Plant Breeding, Bangladesh Agricultural University, Mymensingh-2202, Bangladesh \\ ${ }^{2}$ Bangladesh Institute of Nuclear Agriculture, Mymensingh, Bangladesh \\ *Email: anwargpb@bau.edu.bd
}

\section{ARTICLE HISTORY}

Received: 29 April 2021

Accepted: 22 June 2021

Available online: 14 August 2021

\section{KEYWORDS}

Diallel cross

Heterosis

Combining ability

Gene action

Oil

Fatty acids

Ground nut

\begin{abstract}
Development of a variety having high oil content and desirable fatty acid compositions is a major objective of groundnut (Arachis hypogaea L.) breeding programmes. To study the gene action (through combining ability) and heterosis for oil and fatty acids, an experiment was conducted using a $4 \times 4$ full diallel method. Four parents and their $12 \mathrm{~F}_{1}$ hybrids were evaluated following a randomized complete block design. Data were recorded for oil, fatty acids and oleic-linolenic (O/L) acid ratio. Highly significant genotypic variation was found among the parents and their $F_{1}$ hybrids for the studied traits. The combining ability studies (general, specific and reciprocal) reflected that the oil and fatty acid traits were controlled by both non-additive and additive genes having significant maternal effects. Results also revealed that the parent China Badam was the best general combiner for oil, linolenic acid and $\mathrm{O} / \mathrm{L}$ ratio whereas the parent Binachinabadam-4 for oleic and linoleic acids. Best SCA performance was found from the cross Dacca- $1 \times$ China Badam and Binachinabadam- $4 \times$ China Badam for oil, oleic- and linolenic-acid contents. Significant heterosis for oil content was observed in $F_{1}$ hybrids obtained from the cross Binachinabadam $-4 \times$ China Badam and its reciprocal cross. The cross China Badam $\times$ GC (24)1-1-1 showed a higher $\mathrm{O} / \mathrm{L}$ ratio $(>4)$ along with lower level of saturated fatty acids. Therefore, these crosses could be exploited in future breeding programmes to develop new lines for higher oil and healthy fatty acid compositions.
\end{abstract}

\section{Introduction}

Groundnut (Arachis hypogaea L.), an important oilseed crop in the world, is considered as an important sources of oil, fatty acids, folate, protein and antioxidants (1-3). It is grown in more than 100 countries with a global production of $42.4 \mathrm{Mt}$ from 25.7 Mha of land (2). Groundnut is ranked $4^{\text {th }}$ among the oilseed crops in the world after soybean, rapeseed, and cotton. About 2/3 of the world's total groundnut production is used to produce oil and the remaining $1 / 3$ is used in food products (4). It is the third most important oil seed crop after mustard and sesame in Bangladesh (5). There is a huge shortage in edible oils as Bangladesh requires two Mt of edible oil annually and almost $90 \%$ of the consumption is currently imported. Therefore, consumers and related industries have a growing interest in groundnuts for quality oils and food products.

Groundnut seeds commonly contain 40-50\% edible oil which varies depending on variety, season, and maturity (6). According to one report (7), groundnut processer's benefit can be increased by $7 \%$ through $1 \%$ increase in the seed oil content, indicating greater impact of oil content trait for farmers and traders. Groundnut oil is considered as stable and nutritive as it contains right proportions of saturated and unsaturated fatty acids (also known as healthy fatty acids). Oleic acid, a monounsaturated fatty acid, and linoleic acid, a polyunsaturated fatty acid both retain 75 to $80 \%$ of the total fatty acids in the groundnut oil. A statistical ratio of oleic and linoleic $(\mathrm{O} / \mathrm{L})$ acid in groundnut oil, which ranges from 0.75 to 5.5 or $>2$ imparts stability and improves its shelf life by delaying the development of rancidity (8) that also indicates the oil quality. Saturated fatty acids increases cholesterol in blood and are thus related to heart problems in human. Whereas unsaturated fatty acids are controls cholesterol levels by reducing lowdensity lipoproteins (LDL) and by maintaining highdensity lipoprotein (HDL), boosts heart health, immune system and anti-cancer potential, lowers

C Sikder et al (2021). This is an open-access article distributed under the terms of the Creative Commons Attribution License, which permits unrestricted use, distribution and reproduction in any medium, provided the original author and source are credited (https://creativecommons.org/licenses/by/4.0/). 
blood pressure, prevents cognitive disorders and increase insulin production $(9,10)$. With increasing demand of healthy groundnuts, it is imperative to enhance its unsaturated fatty acid contents to increase its acceptance and use.

For improving oil content and quality related traits in groundnut, knowledge of genetic control of these traits regulating oils and fatty acids composition and genetic variation created through hybridization is necessary. Although inheritance of the oil and fatty acid traits has been reported, the mechanism of the inheritance of these traits may be different due to the differences in the parental sources. Previous research on fatty acids in groundnut has been suggested that the inheritance is governed by both additive and non-additive gene actions $(11,12)$. Diallel crosses and combining ability studies provides an opportunity to know the mode of inheritance and provide a clear concept for breeders to understand the basis on which certain parental traits could be exploited in the breeding programme. Additionally, general combining ability (GCA), specific combining ability (SCA) variance provides breeders an insight on additive and non-additive inheritance, respectively where reciprocal combining ability (RCA) signify the maternal effect (13). Heterosis study also helps the breeder to assess the superiority and inferiority of the $F_{1}$ hybrids as compared to their parents and also for selecting suitable parents for achieving higher genetic gain. Considering the above facts, the present research was conducted to know the gene action controlling oil content and fatty acid compositions through combining ability and heterosis analyses that would help the breeder to select parents or superior lines and to set an appropriate breeding program to increase groundnut oil content as well as to improve the oil quality conferring healthy fatty acid compositions.

\section{Materials and Methods}

\section{Experimental site and breeding material}

The experiment was conducted at the field experimental plot of Bangladesh Institute of Nuclear Agriculture (BINA), Mymensingh, Bangladesh during the period of $2018-2020$ using a $4 \times 4$ intra-specific $F_{1}$ diallel cross of groundnut. Breeding material comprised of a set of four groundnut genotypes viz., Binachinabadam-4, Dacca-1, GC (24)-1-1-1 and China Badam, having diverse origin. Phenotype and other details of the parental lines used in the study are mentioned in Table 1 and Fig. 1. All genotypes were
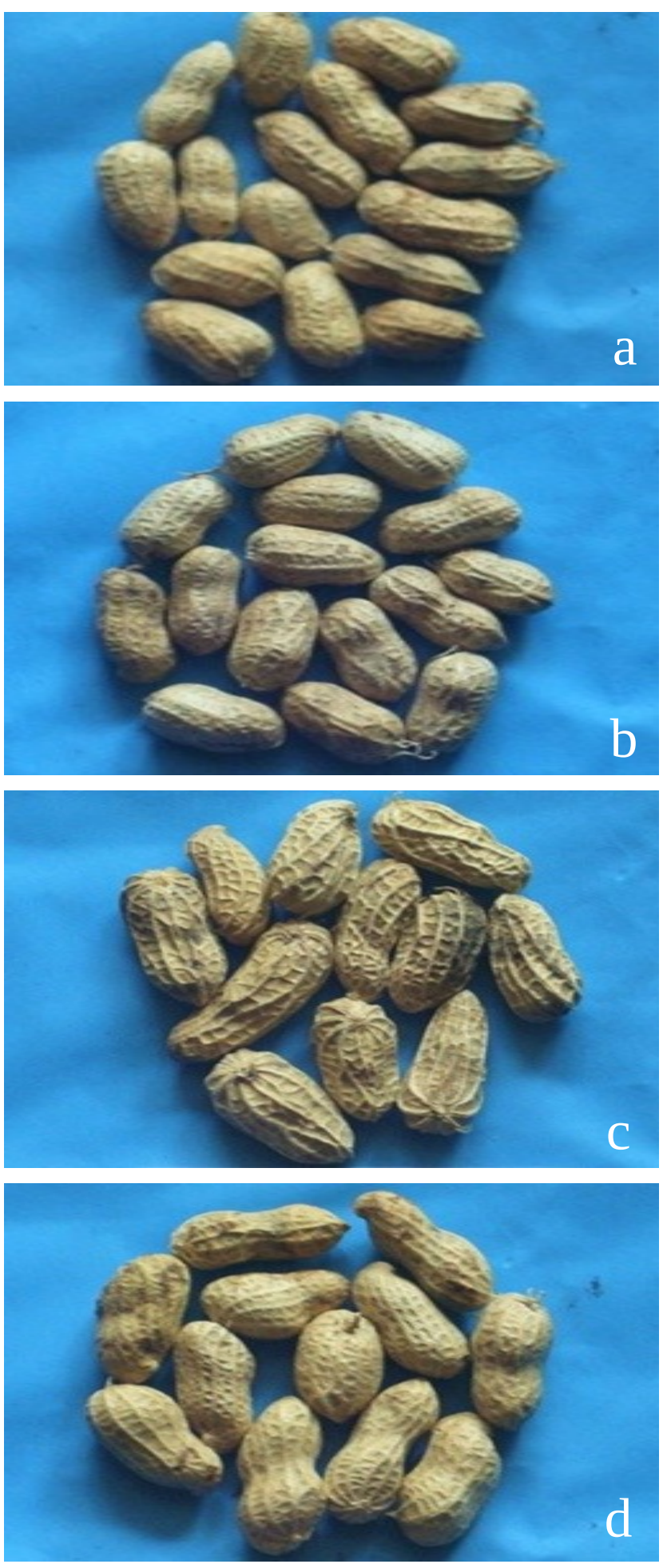

Fig. 1. Phenotypic appearance of four parents (a) Binachinabadam-4, (b) Dacca-1, (c) GC (24)-1-1-1, (d) China Badam used in the $4 \times 4$ full diallel crossing experiment.

Table 1. Groundnut parents used in the study along with different attributes

\begin{tabular}{|c|c|c|c|c|c|c|c|}
\hline Varieties/genotypes & Source & $\begin{array}{c}\text { Botanical } \\
\text { type }\end{array}$ & $\begin{array}{l}\text { Flowering and } \\
\text { maturity date }\end{array}$ & Plant height & $\begin{array}{l}\text { Seed size and } \\
\text { shape }\end{array}$ & $\begin{array}{c}\text { 100-seed } \\
\text { weight (gm) }\end{array}$ & Oil content $\%$ \\
\hline Binachinabadam-4 & $\begin{array}{l}\text { Plant Breeding } \\
\text { Division, BINA }\end{array}$ & Spanish & Relatively late & $40-60 \mathrm{~cm}$ & $\begin{array}{l}\text { Small and } \\
\text { round }\end{array}$ & $36-42$ & 47 \\
\hline Dacca-1 & $\begin{array}{l}\text { Plant Breeding } \\
\text { Division, BINA }\end{array}$ & Spanish & Relatively late & $40-60 \mathrm{~cm}$ & $\begin{array}{l}\text { Small and } \\
\text { round }\end{array}$ & $36-42$ & 46 \\
\hline GC (24)-1-1-1 & $\begin{array}{l}\text { Plant Breeding } \\
\text { Division, BINA }\end{array}$ & Valencia & Relatively early & $35-50 \mathrm{~cm}$ & $\begin{array}{c}\text { Medium and } \\
\text { elongated }\end{array}$ & $40-50 \mathrm{~g}$ & 48 \\
\hline China Badam & $\begin{array}{l}\text { Plant Breeding } \\
\text { Division, BINA }\end{array}$ & Valencia & Relatively early & $35-50 \mathrm{~cm}$ & $\begin{array}{l}\text { Large and } \\
\text { elongated }\end{array}$ & $40-50 \mathrm{~g}$ & 49 \\
\hline
\end{tabular}


crossed in complete diallel fashion during the winter of 2018-2019. During 2019-2020, a set of four parents and their $12 \mathrm{~F}_{1}$ hybrids (6 direct and 6 reciprocal crosses) were evaluated in a randomized complete block design with three replications. Each plot was designed $1.5 \mathrm{~m} \times 5 \mathrm{~m}$ in size, consisting of sixteen rows with row length of $1.5 \mathrm{~m}$. Plant to plant and row to row distance were 20 and $30 \mathrm{~cm}$, respectively. All recommended cultural practices and inputs including thinning, hoeing, irrigation and pest control were carried out using the standard procedures.

\section{Determination of oil content}

Oil content was determined using Soxhlet method (14) with minor modifications. Two gram of oven dried groundnut seeds of each genotype were weighed and pulverized into fine powder with a mortar and pestle. Then the groundnut meal was extracted with petroleum benzene for $17 \mathrm{hrs}$ in Soxhlet apparatus. Powder weight before and after extraction was taken, the difference between the two weights was expressed in terms of oil percentage. The advantage of using Soxhlet extraction is that the solvent used in this method penetrates faster to the kernel powder, dissolve oil in the solvent and make a complete extraction. Additionally, this method is very efficient, quick, requires less solvent and convenient for automation and is more acceptable than other extraction methods.

\section{Determination of fatty acids}

Oil content was analysed for fatty acids through gas chromatography (using a VARIAN, CP-3800 Gas Chromatograph) in Bangladesh Institute of Nuclear Agriculture (BINA), Mymensingh with a flame ionization detector (FID) following the slight modification of the protocol described (15). First, the groundnut oil was converted into fatty acid methyl ester (FAME) which was then injected to the GC machine with FID and different types of peaks with retention time was observed. The observed retention time was compared with the standard FAME (Supelco 37 component FAME mix, CRM47885) to confirm the specific fatty acid presence in the oil.

\section{Data recording and statistical analysis}

Data were recorded on oil content (\%), unsaturated fatty acid (oleic acid, linoleic, linolenic, palmitoleic acid) and saturated fatty acid (lauric, myristic, palmitic, stearic, arachidic acid) compositions and olic/linoleic acid ratio (O/L). The data were subjected to analysis of variance (ANOVA) valid for RCBD design. ANOVA, mean, combining ability and heterosis were calculated using $\mathrm{PB}$ tools version: 1.3 of IRRI (16) and Minitab-17 software. Mean separation was done following Duncan's Multiple Range Test (DMRT) at 5\% level of probability. Standard Model 1 was used for combining ability
(GCA, SCA and RCA) analysis for each of the trait (17). The amount of heterosis for a particular trait was calculated by comparing the mean of $F_{1}$ hybrids over mid-parental value of the traits following standard formula (18).

\section{Results}

The results of analysis of variance showed highly significant genotypic differences $(P \leq 0.01)$ for oil content and fatty acid compositions among the parents except for oleic/linoleic $(\mathrm{O} / \mathrm{L})$ ratio (Table 2$)$.

\section{oil Content and fatty acid composition in the parents and hybrids}

The oil content and fatty acid compositions in the parents and $F_{1}$ hybrids of groundnut that were obtained from a $4 \times 4$ diallel crossing experiment are presented in Table 2. In respect of oil content, the parent Dacca-1 showed the highest percentage of oil content (51.73\%) whereas Binachinabadam- 4 showed the lowest $(47.33 \%)$ content of oil (Table 3$)$. The cross combination Binachinabadam- 4 China Badam was the highest oil producer $(55.40 \%)$ followed by the cross Dacca-1 $\times$ China Badam (54.60\%) (Table 3). The highest oleic acid was recorded in Binachinabadam-4 (34.60\%) whereas the lowest content (21.73\%) was recorded in Dacca-1. Among the crosses and reciprocal crosses, GC (24)-1-1-1 × Binachinabadam-4 showed the highest (33.67\%) whereas GC (24)-1-1-1 $\times$ China Badam showed the lowest $(14.90 \%)$ oleic acid content. The parent Binachinabadam-4 showed the highest (36.69\%) content of linoleic acid. The cross combination Binachinabadam-4 $\times$ Dacca-1 showed the highest (28.66\%) linoleic acid content whereas the parent Cina Badam and the cross China Badam $\times$ GC (24)-1-1-1showed the lowest content (22.14 and 7.11\%, respectively) of linolenic acid (Table 3 ). The highest value (1.54) for O/L ratio was found for the genotype Cina Badam whereas the highest O/L ratio (4.87) was found from the cross China Badam $\times$ GC (24)-1-1-1. The highest linolenic acid content (28.05\%) was found in the parents GC (24)-1-1-1 whereas the lowest $(1.26 \%)$ was found in the genotype Binachinabadam4. Similarly, the highest (47.53\%) linolenic acid was found from the cross Dacca- $1 \times$ China Badam, however, it showed a non-significant difference with cross China Badam $\times$ GC (24)-1-1-1(45.39\%). The lowest content of linolenic content was found from the cross GC (24)-1-1-1 × Binachinabadam-4. The cross combination Dacca-1 $\times$ Binachinabadam-4 showed the highest (13.59\%) palmitoleic acid. Importantly, it's reciprocal cross Binachinabadam- $4 \times$ Dacca- 1 showed the lowest (3.49\%) amount of palmitoleic acid which showed a non-significant difference with the parent Dacca-1 (5.39\%) and the cross combination China badam $\times$ GC (24)-1-1-1 (6.07\%) (Table 3). The parent

Table 2. Analysis of variance for oil content and fatty acid compositions in a $4 \times 4$ diallel crossing experiment of groundnut

\begin{tabular}{|c|c|c|c|c|c|c|c|c|c|c|c|c|}
\hline Item & df & Oil (\%) & $\begin{array}{l}\text { Oleic } \\
\text { acid }\end{array}$ & $\begin{array}{c}\text { Linoleic } \\
\text { acid }\end{array}$ & $\begin{array}{c}\mathrm{O} / \mathrm{L} \\
\text { ratio }\end{array}$ & $\begin{array}{c}\text { Linolenic } \\
\text { acid }\end{array}$ & $\begin{array}{c}\text { Palmitoleic } \\
\text { acid }\end{array}$ & $\begin{array}{c}\text { Lauric } \\
\text { acid }\end{array}$ & $\begin{array}{l}\text { Myristic } \\
\text { acid }\end{array}$ & $\begin{array}{c}\text { Palmitic } \\
\text { acid }\end{array}$ & $\begin{array}{c}\text { Stearic } \\
\text { acid }\end{array}$ & $\begin{array}{c}\text { Arachidic } \\
\text { acid }\end{array}$ \\
\hline Replication & 2 & 0.27 & 8.47 & 39.47 & 1.32 & 4.91 & 1.33 & 1.04 & 1.95 & 0.91 & 2.66 & 0.94 \\
\hline Genotype & 15 & $18.72^{* *}$ & $84.34^{* *}$ & $173.71^{* *}$ & 3.65 & $483.68^{* *}$ & $11.52^{* *}$ & $31.13^{* *}$ & $10.44^{* *}$ & $24.23^{* *}$ & $7.67^{* *}$ & $9.17^{* *}$ \\
\hline Error & 30 & 0.39 & 8.83 & 7.62 & 0.38 & 5.06 & 1.68 & 0.20 & 0.22 & 1.91 & 0.57 & 0.72 \\
\hline
\end{tabular}


Table 3. Oil and fatty acid contents in the parents and $F_{1}$ hybrids obtained from a $4 \times 4$ diallel crossing experiment of groundnut

\begin{tabular}{|c|c|c|c|c|c|c|c|c|c|c|c|}
\hline Parents & Oil (\%) & $\begin{array}{l}\text { Oleic acid } \\
\text { (\%) }\end{array}$ & $\begin{array}{l}\text { Linoleic } \\
\text { acid (\%) }\end{array}$ & $\begin{array}{l}\mathrm{O} / \mathrm{L} \\
\text { ratio }\end{array}$ & $\begin{array}{l}\text { Linolenic } \\
\text { acid (\%) }\end{array}$ & $\begin{array}{l}\text { Palmitoleic } \\
\text { acid (\%) }\end{array}$ & $\begin{array}{r}\text { Lauric } \\
\text { acid (\%) }\end{array}$ & $\begin{array}{l}\text { Myristic } \\
\text { acid (\% }\end{array}$ & $\begin{array}{l}\text { ic Palmitic } \\
\text { o) acid (\%) }\end{array}$ & $\begin{array}{l}\text { c Stearic } \\
\text { acid (\%) }\end{array}$ & $\begin{array}{l}\text { Arachidic } \\
\text { acid (\%) }\end{array}$ \\
\hline Binachinabadam-4 & $47.33 \mathrm{hi}$ & $34.60 \mathrm{a}$ & $36.69 \mathrm{a}$ & $0.95 \mathrm{f}$ & $1.26 \mathrm{i}$ & $9.15 \mathrm{~b}$ & $0.63 \mathrm{ef}$ & $1.48 \mathrm{~d}$ & \multicolumn{2}{|c|}{11.59 a 5.26 a } & $1.64 \mathrm{e}$ \\
\hline Dacca-1 & $51.73 \mathrm{de}$ & $21.73 \mathrm{~d}$ & $28.66 \mathrm{~b}$ & $0.84 \mathrm{f}$ & $11.74 \mathrm{~h}$ & $5.39 \mathrm{de}$ & 12.81 & a6.25 a & \multicolumn{2}{|c|}{$12.52 \mathrm{a} 0.89 \mathrm{fg}$} & $1.56 \mathrm{f}$ \\
\hline GC (24)-1-1-1 & $49.83 \mathrm{f}$ & $28.02 \mathrm{bc}$ & $26.40 \mathrm{bc}$ & $1.06 \mathrm{~d}-\mathrm{f}$ & $28.05 \mathrm{f}$ & $7.93 \mathrm{bd}$ & $0.14 \mathrm{f}$ & $0.62 \mathrm{efg}$ & \multicolumn{2}{|c|}{7.64 c-1.21 e-g } & $0.35 \mathrm{ij}$ \\
\hline China Badam & $49.00 \mathrm{fg}$ & 33.69 a & 22.14 c-e & $1.54 \mathrm{c}-\mathrm{f}$ & $21.48 \mathrm{~g}$ & $6.85 \mathrm{~b}-\mathrm{d}$ & $5.03 \mathrm{~b}$ & $3.87 \mathrm{c}$ & \multicolumn{2}{|c|}{$8.53 \mathrm{~b}$ - 1.35 e-g } & $0.51 \mathrm{hi}$ \\
\hline \multicolumn{12}{|l|}{ Crosses } \\
\hline $\begin{array}{l}\text { Binachinabadam-4 } \\
\text { Dacca-1 }\end{array}$ & $49.30 \mathrm{f}$ & $28.88 \mathrm{bc}$ & $28.66 \mathrm{~b}$ & $1.98 \mathrm{f}$ & 36.96 de & $3.49 \mathrm{e}$ & $3.03 \mathrm{~d}$ & $3.56 \mathrm{c}$ & $6.46 \mathrm{c}-\mathrm{f} \quad 3$ & $3.71 \mathrm{bc}$ & $0.75 \mathrm{gh}$ \\
\hline $\begin{array}{l}\text { Binachinabadam- } \\
4 \times G C(24)-1-1-1\end{array}$ & $52.30 \mathrm{~d}$ & 28.36 bc & $26.40 \mathrm{bc}$ & $1.28 \mathrm{c}-\mathrm{f}$ & $26.48 \mathrm{f}$ & $8.36 \mathrm{bc}$ & $1.06 \mathrm{e}$ & $1.16 \mathrm{de}$ & $8.69 \mathrm{bc}$ & $1.87 \mathrm{e}-\mathrm{g}$ & $1.39 \mathrm{~d}$ \\
\hline $\begin{array}{l}\text { Binachinabadam- } \\
4 \times \text { China Badam }\end{array}$ & $55.40 \mathrm{a}$ & $27.28 \mathrm{c}$ & $23.14 \mathrm{~cd}$ & $1.26 \mathrm{c}-\mathrm{f}$ & $28.68 \mathrm{f}$ & $7.48 \mathrm{~b}-\mathrm{d}$ & 0.76 ef & $0.86 \mathrm{~d}-\mathrm{g}$ & $8.32 \mathrm{~b}-\mathrm{d} \quad 3$ & $3.13 \mathrm{~cd}$ & $2.35 \mathrm{c}$ \\
\hline $\begin{array}{l}\text { Dacca-1× } \\
\text { Binachinabadam- } 4\end{array}$ & $50.96 \mathrm{e}$ & $19.96 \mathrm{~d}$ & $18.68 \mathrm{e}-\mathrm{g}$ & $1.19 \mathrm{~d}-\mathrm{f}$ & $25.57 \mathrm{f}$ & $13.59 \mathrm{a}$ & $3.80 \mathrm{c}$ & $5.01 \mathrm{~b}$ & $10.44 \mathrm{ab} \quad 3$ & $3.30 \mathrm{~cd}$ & $2.16 \mathrm{~cd}$ \\
\hline $\begin{array}{l}\text { Dacca-1 } \\
\times \text { GC (24)-1-1-1 }\end{array}$ & $46.93 \mathrm{i}$ & $27.01 \mathrm{c}$ & $19.48 \mathrm{~d}-\mathrm{f}$ & $1.41 \mathrm{c}-\mathrm{f}$ & $35.41 \mathrm{e}$ & $8.65 \mathrm{bc}$ & $0.77 \mathrm{ef}$ & $0.18 \mathrm{~g}$ & $6.35 \mathrm{~d}-\mathrm{g} \quad 2$ & $2.11 \mathrm{~d}-\mathrm{f}$ & $2.69 \mathrm{c}$ \\
\hline $\begin{array}{l}\text { Dacca-1 } \\
\times \text { China Badam }\end{array}$ & $54.60 \mathrm{~b}$ & $26.92 \mathrm{c}$ & $10.90 \mathrm{hi}$ & $2.26 \mathrm{c}$ & $47.53 \mathrm{a}$ & $8.04 \mathrm{~b}-\mathrm{d}$ & $0.24 \mathrm{f}$ & $0.34 \mathrm{fg}$ & $5.02 \mathrm{fg}$ & $0.68 \mathrm{~g}$ & $0.41 \mathrm{ij}$ \\
\hline $\begin{array}{l}\text { GC (24)-1-1-1 } \\
\times \text { Binachinabadam-4 }\end{array}$ & $52.43 \mathrm{bc}$ & $33.67 \mathrm{a}$ & $23.14 \mathrm{~cd}$ & $1.58 \mathrm{c}-\mathrm{f}$ & $21.21 \mathrm{~g}$ & $7.65 \mathrm{bd}$ & 0.70 ef & $0.69 \mathrm{~d}-\mathrm{f}$ & $11.17 \mathrm{a}$ & $1.73 \mathrm{e}-\mathrm{g}$ & $1.22 \mathrm{f}$ \\
\hline $\begin{array}{l}\text { GC (24)-1-1-1 } \\
\times \text { Dacca-1 }\end{array}$ & $48.00 \mathrm{gh}$ & $27.51 \mathrm{bc}$ & $14.44 \mathrm{gh}$ & $1.91 \mathrm{c}-\mathrm{e}$ & $41.48 \mathrm{bc}$ & $9.15 \mathrm{~b}$ & $0.19 \mathrm{f}$ & $0.20 \mathrm{~g}$ & $5.33 \mathrm{fg} \quad 2$ & $2.15 \mathrm{de}$ & $2.85 \mathrm{~b}$ \\
\hline $\begin{array}{l}\text { GC }(24)-1-1-1 \times \text { China } \\
\text { Badam }\end{array}$ & 51.46 de & $14.90 \mathrm{e}$ & $22.23 \mathrm{c}-\mathrm{e}$ & $0.70 \mathrm{f}$ & $39.95 \mathrm{~cd}$ & $8.27 \mathrm{bc}$ & $0.21 \mathrm{f}$ & $1.03 \mathrm{~d}-\mathrm{f}$ & $5.53 \mathrm{e}-\mathrm{g} \quad 4$ & $4.83 \mathrm{ab}$ & $0.86 \mathrm{~g}$ \\
\hline $\begin{array}{l}\text { China Badam } \\
\times \text { Binachinabadam-4 }\end{array}$ & $53.46 \mathrm{~b}$ & $32.40 \mathrm{ab}$ & $16.34 \mathrm{fg}$ & $2.00 \mathrm{~cd}$ & $27.56 \mathrm{f}$ & $7.10 \mathrm{~b}-\mathrm{d}$ & $1.19 \mathrm{e}$ & $1.31 \mathrm{de}$ & $12.71 \mathrm{a}$ & $2.02 \mathrm{ef}$ & $0.25 \mathrm{j}$ \\
\hline $\begin{array}{l}\text { China Badam } \\
\times \text { Dacca-1 }\end{array}$ & $53.63 \mathrm{~b}$ & 30.68 ac & $9.00 \mathrm{i}$ & $3.79 \mathrm{~b}$ & $44.62 \mathrm{ab}$ & $6.94 \mathrm{~b}-\mathrm{d}$ & 0.71 ef & $0.21 \mathrm{~g}$ & $5.43 \mathrm{e}-\mathrm{g}$ & $5.07 \mathrm{a}$ & $3.36 \mathrm{a}$ \\
\hline $\begin{array}{l}\text { China Badam } \\
\times \text { GC (24)-1-1-1 }\end{array}$ & 51.46 de & $30.92 \mathrm{ac}$ & $7.11 \mathrm{i}$ & $4.87 \mathrm{a}$ & $45.39 \mathrm{a}$ & $6.07 \mathrm{c}-\mathrm{e}$ & $0.44 \mathrm{ef}$ & $1.31 \mathrm{de}$ & $4.12 \mathrm{~g}$ & $5.13 \mathrm{a}$ & $0.83 \mathrm{~g}$ \\
\hline Min. & 46.80 & 6.40 & 4.40 & 0.40 & 0.98 & 3.10 & 0.10 & 0.10 & 3.24 & 0.16 & 1.89 \\
\hline Max. & 55.70 & 38.10 & 39.08 & 5.11 & 50.25 & 14.44 & 13.76 & 7.22 & 14.22 & 7.56 & 34.76 \\
\hline
\end{tabular}

Note: Different letters in a column showed significant difference at 5\% level of probability following DMRT test

Dacca-1 had the highest percentage of lauric acid (12.81\%), myristic acid (6.25\%) and palmitic acid $(12.52 \%)$ while the cross combination Binachinabadam-4 × Dacca-1 showed the highest content of myristic acid and its reciprocal cross (Dacca-1 $\times$ Binachinabadam-4) showed the highest content of lauric acid. Similarly, the cross combination China Badam $\times$ Binachinabadam -4 had significantly higher percentage of palmitic acid (12.71\%) which showed a non-significant difference with the parent Dacca-1, and the cross GC (24)-1-1-1 × Binachinabadam-4 (Table 3). The parent Binachinabadam-4 showed the highest content of stearic and arachidic acid (5.26 and 1.64\%, respectively) whereas the cross combinations China Badam $\times$ GC (24)-1-1-1 and China Badam $\times$ Dacca-1 showed the highest content of stearic acid. The cross combination China Badam $\times$ Binachinabadam-4 showed the lowest content $(0.25 \%)$ of arachidic acid (Table 3).

\section{Analysis of variance for combining ability}

The mean squares for GCA, SCA and RCA were highly significant for all traits including O/L ratio (Table 4). The variance due to dominance deviation $\left(V_{D}\right)$ for oils and all fatty acids was much higher than those of the additive deviation $\left(V_{A}\right)$ (Table 4$)$.

\section{Combining ability of parents}

The parent China Badam showed the best general combining effect for oil content while the other three parents exhibited negative GCA effects, however, GC (24)-1-1-1 being the poorest (Table 5). Binachinabadam-4 was a good general combiner for oleic, linoleic, palmitic, and stearic acid. In contrast, Dacca-1 was a good general combiner for lauric, myristic and arachidic acid contents. Similarly, GC (24)-1-1-1 was a good combiner for linolenic acid and China Badam for linolenic acid and O/L (Table 5). China Badam was the only parent which showed significant positive GCA for $\mathrm{O} / \mathrm{L}$ ratio whereas Binachinabadam-4 showed high negative nonsignificant effect followed by other parental genotypes GC (24)- 1-1-1 and Dacca-1.

For oil content, four cross combinations viz., Binachinabadam-4 × GC (24)-1-1-1, Binachinabadam$4 \times$ China Badam, Dacca-1 $\times$ China Badam and GC (24)-1-1-1 $\times$ China Badam showed significant positive SCA effects (Table 6). In contrast, different types of fatty acids content mostly showed significant negative to non-significant positive SCA effects. Besides, few cross-combinations showed significant positive SCA effect i.e., Binachinabadam-4 $\times$ Dacca-1 for linolenic acid and myristic acid; Binachinabadam$4 \times$ GC (24)-1-1-1 for lauric acid; Dacca-1 × GC (24)-1-11 for linolenic and arachidic acid; Dacca- $1 \times$ China Badam for linolenic, arachidic acid and O/L ratio and GC (24)-1-1-1 × China Badam for linolenic acid and $\mathrm{O} / \mathrm{L}$ ratio (Table 6).

Based on the results of RCA effect, the cross combination Dacca- $1 \times$ Binachinabadam-4 showed significant positive RCA effects for oleic and linolenic 
Table 4. Analysis of variance for combining ability of oils and fatty acid compositions in a $4 \times 4$ diallel crossing experiment of groundnut

\begin{tabular}{|c|c|c|c|c|c|c|c|c|c|c|c|c|}
\hline \multirow{2}{*}{$\begin{array}{l}\text { Source of } \\
\text { Variation }\end{array}$} & \multirow[b]{2}{*}{ df } & \multicolumn{11}{|c|}{ MS } \\
\hline & & Oil & $\begin{array}{l}\text { Oleic } \\
\text { acid }\end{array}$ & $\begin{array}{l}\text { Linoleic } \\
\text { acid }\end{array}$ & O/L ratio & $\begin{array}{c}\text { Linolenic } \\
\text { acid }\end{array}$ & $\begin{array}{c}\text { Palmitoleic } \\
\text { acid }\end{array}$ & $\begin{array}{c}\text { Lauric } \\
\text { acid }\end{array}$ & $\begin{array}{l}\text { Myristic } \\
\text { acid }\end{array}$ & $\begin{array}{l}\text { Palmitic } \\
\text { acid }\end{array}$ & $\begin{array}{c}\text { Stearic } \\
\text { acid }\end{array}$ & $\begin{array}{l}\text { Arachidic } \\
\text { acid }\end{array}$ \\
\hline GCA & 3 & $6.54^{* *}$ & $29.35^{* *}$ & $80.11^{* *}$ & $1.01^{* *}$ & $303.44^{* *}$ & $1.70^{* *}$ & $21.33^{* *}$ & $5.59 * *$ & $15.64^{* *}$ & $1.91^{* *}$ & $4.85^{* *}$ \\
\hline SCA & 6 & $11.41^{* *}$ & $22.49^{* *}$ & $79.08^{* *}$ & 0.08 & $231.67^{* *}$ & $1.41^{* *}$ & $15.14^{* *}$ & $5.69 * *$ & $8.66^{* *}$ & $4.62^{* *}$ & $7.53^{* *}$ \\
\hline RCA & 6 & $0.42^{* *}$ & $33.11^{* *}$ & $25.61^{* *}$ & $1.36^{* *}$ & $19.46^{* *}$ & $9.06^{* *}$ & $0.12^{* *}$ & $0.22^{* *}$ & $3.70^{* *}$ & $1.68^{* *}$ & $1.16^{* *}$ \\
\hline Error & 30 & 0.13 & 2.93 & 2.34 & 0.12 & 1.69 & 0.86 & 0.06 & 0.07 & 0.61 & 0.14 & 0.07 \\
\hline$V_{A}$ & & 0.09 & 4.18 & 3.46 & 0.12 & 44.64 & 0.16 & 3.67 & 0.17 & 3.80 & 0.21 & 0.27 \\
\hline$V_{D}$ & & 30.10 & 48.14 & 188.91 & 1.67 & 566.61 & 1.36 & 37.11 & 13.61 & 19.81 & 8.24 & 18.36 \\
\hline
\end{tabular}

Note: $^{*}$ and ${ }^{* *}$ indicates significant at $5 \%$ and $1 \%$ level of probability, respectively

Table 5. General combining ability effects for oils and fatty acid compositions of parents in a $4 \times 4$ diallel crossing experiment of groundnut

\begin{tabular}{|c|c|c|c|c|c|c|c|c|c|c|c|}
\hline Character Parent & Oil (\%) & $\begin{array}{l}\text { Oleic acid } \\
\text { (\%) }\end{array}$ & $\begin{array}{l}\text { Linoleic } \\
\text { acid (\%) }\end{array}$ & $0 / \mathrm{L}$ ratio & $\begin{array}{l}\text { Linolenic } \\
\text { acid (\%) }\end{array}$ & $\begin{array}{l}\text { Palmitoleic } \\
\text { acid (\%) }\end{array}$ & $\begin{array}{c}\text { Lauric } \\
\text { acid (\%) }\end{array}$ & $\begin{array}{l}\text { Myristic } \\
\text { acid (\%) }\end{array}$ & $\begin{array}{l}\text { Palmitic } \\
\text { acid (\%) }\end{array}$ & $\begin{array}{c}\text { Stearic } \\
\text { acid (\%) }\end{array}$ & $\begin{array}{l}\text { Arachidic } \\
\text { acid (\%) }\end{array}$ \\
\hline Binachinabadam-4 & -0.11 & $2.03^{* *}$ & $4.15^{* *}$ & -0.39 & $-9.08^{* *}$ & 0.37 & $-0.50^{* *}$ & 0.18 & $2.00^{* *}$ & $0.62^{* *}$ & 0.98 \\
\hline Dacca-1 & -0.06 & $-2.38 * *$ & -1.59 & -0.02 & 1.67 & -0.11 & $2.31^{* *}$ & $0.99^{* *}$ & -0.10 & -0.37 & $2.98^{* *}$ \\
\hline GC (24)-1-1-1 & $-0.91^{* *}$ & -0.57 & 0.58 & -0.06 & $3.04^{* *}$ & 0.29 & $-1.52^{* *}$ & -0.01 & -1.05 & -0.31 & $-1.51^{*}$ \\
\hline China Badam & $2.10^{* *}$ & 0.92 & $-3.15^{* *}$ & $0.46^{* *}$ & $4.34^{* *}$ & $-0.49 * *$ & -0.28 & -0.15 & -0.84 & 0.28 & $-2.46^{* *}$ \\
\hline SE (gi) & 0.14 & 0.52 & 0.46 & 0.11 & 0.40 & 0.10 & 0.08 & 0.08 & 0.24 & 0.12 & 0.27 \\
\hline
\end{tabular}

Note: * and ** indicates significant at $5 \%$ and $1 \%$ level of probability, respectively

acid, GC (24)-1-1-1 × Binachinabadam-4 for arachidic acid, China Badam $\times$ Binachinabadam 4 for oil content and archidic acid, China Badam $\times$ GC (24)-1-11 for linoleic and O/L ratio (Table 7). Rest of the traits showed non-significant positive or non-significant negative or significant negative RCA effects in every cross-combination (Table 7).

\section{Heterosis for oil and fatty acids content}

For oil content, most of the crosses showed significant positive mid-parent heterosis however the highest significant positive heterosis $(15.04 \%)$ was found from the cross Binachinabadam- $4 \times$ China Badam and the highest significant negative $(-7.58 \%)$ heterosis was found in the cross combination Dacca-1 $\times$ GC (24)-1-1-1 (Table 8). The cross combination GC (24)-1-1-1 $\times$ Binachinabadam-4 showed the highest (61.31\%) significant positive heterosis for oleic acid however the same cross showed a non-significant positive heterosis (10.05\%) for linoleic acid content. In case of O/L ratio, most of the crosses showed nonsignificant positive heterosis whereas the cross combination China Badam $\times$ GC (24)-1-1-1 showed the highest significant positive heterosis (25.64\%) followed by the cross combination China Badam $\times$ Dacca-1 and its reciprocal cross Dacca- $1 \times$ China Badam. In addition, the cross combination Binachinabadam-4 $\times$ Dacca-1 showed the highest significant positive $(468.43 \%)$ heterosis for linolenic acid and the cross combination GC (24)-1-1-1 × China Badam showed the minimum significant positive heterosis (61.30\%). In case of plamitoleic acid, the cross combination Dacca-1 $\times$ Binachinabadam- 4 showed the highest significant positive heterosis (81.59\%). Similarly, the cross combination Binachinabadam $-4 \times$ Dacca- 1 showed significant positive heterosis for lauric, myristic and palmitic acid (323.61, 65.93 and 35.63\%, respectively). Additionally, the cross combination China Badam $\times$ GC (24)-1-1-1 showed the highest significant positive mid-parent heterosis for stearic and arachidic acid (299.48 and 134.34\%, respectively) (Table 8).

\section{Discussion}

The knowledge on combining ability and type of gene action responsible for the regulation of expression of different traits is important for planning appropriate breeding strategies. Diallel cross has been extensively used for analyses of GCA, SCA, RCA and heterosis. In this research, an attempt has been made to explore the combining abilities of oil and fatty acids content in a $4 \times 4$ full diallel crosses of groundnut. A significant variation was found for oil content as well as fatty acid compositions among the parents and their hybrids. Similar to our results, significant genotypic differences among parents and their $F_{1}$

Table 6. Specific combining ability effects for oils and fatty acid compositions of cross combinations in a $4 \times 4$ diallel crossing experiment of groundnut

\begin{tabular}{|c|c|c|c|c|c|c|c|c|c|c|c|}
\hline Character X Crosses & $\begin{array}{l}\text { Oil } \\
\text { (\%) }\end{array}$ & $\begin{array}{c}\text { Oleic } \\
\text { acid (\%) }\end{array}$ & $\begin{array}{l}\text { Linoleic } \\
\text { acid (\%) }\end{array}$ & $\begin{array}{c}\mathrm{O} / \mathrm{L} \\
\text { ratio }\end{array}$ & $\begin{array}{l}\text { Linolenic } \\
\text { acid (\%) }\end{array}$ & $\begin{array}{l}\text { Palmitoleic } \\
\text { acid (\%) }\end{array}$ & $\begin{array}{c}\text { Lauric } \\
\operatorname{acid}(\%)\end{array}$ & $\begin{array}{l}\text { Myristic } \\
\text { acid (\%) }\end{array}$ & $\begin{array}{l}\text { Palmitic } \\
\text { acid }(\%)\end{array}$ & $\begin{array}{c}\text { Stearic } \\
\text { acid }(\%)\end{array}$ & $\begin{array}{c}\text { Arachidic } \\
\text { Acid (\%) }\end{array}$ \\
\hline Binachinabadam- $4 \times$ Dacca-1 & $-0.78^{*}$ & $-3.33^{* *}$ & $-5.65^{* *}$ & 0.21 & $8.47^{* *}$ & 0.41 & -0.38 & $1.33^{* *}$ & -1.56 & 0.54 & $-0.41^{* *}$ \\
\hline $\begin{array}{l}\text { Binachinabadam-4 } \\
\times \text { GC (24)-1-1-1 }\end{array}$ & $2.10^{* *}$ & 1.68 & -1.60 & 0.04 & -0.31 & -0.63 & $0.94^{* *}$ & 0.01 & 0.89 & $-1.25^{* *}$ & 0.16 \\
\hline $\begin{array}{l}\text { Binachinabadam- } 4 \\
\times \text { China Badam }\end{array}$ & $2.26^{* *}$ & -1.00 & -1.51 & -0.22 & 2.62 & -0.25 & -0.22 & $-0.70^{* *}$ & 1.23 & $-0.82^{* *}$ & 0.29 \\
\hline Dacca-1 × GC (24)-1-1-1 & $-3.53^{* *}$ & 2.34 & -1.65 & -0.04 & $3.52^{* *}$ & 0.89 & $-2.29 * *$ & $-1.53^{* *}$ & -1.11 & 0.04 & $0.93^{* *}$ \\
\hline Dacca-1 × China Badam & $1.10^{* *}$ & 2.37 & $-4.93^{* *}$ & $0.79 *$ & $9.82^{* *}$ & 0.74 & $-3.53^{* *}$ & $-2.31^{* *}$ & $-1.94^{* *}$ & 0.54 & $0.39 *$ \\
\hline GC (24)-1-1-1×China Badam & $1.10^{* *}$ & $-5.32^{* *}$ & -2.38 & $0.60^{*}$ & $5.04^{* *}$ & $-1.22^{* *}$ & 0.15 & 0.60 & -1.39 & $1.26^{* *}$ & -0.23 \\
\hline SE (sij) & 0.20 & 0.94 & 0.83 & 0.19 & 0.72 & 0.26 & 0.14 & 0.15 & 0.44 & 0.24 & 0.04 \\
\hline
\end{tabular}

Note: *and ${ }^{* *}$ indicates significant at $5 \%$ and $1 \%$ level of probability, respectively 
Table 7. Reciprocal combining ability effects for oil and fatty acid compositions of cross combinations in a $4 \times 4$ diallel crossing experiment of groundnut

\begin{tabular}{|c|c|c|c|c|c|c|c|c|c|c|c|}
\hline Character x Crosses & $\begin{array}{l}\text { Oil } \\
(\%)\end{array}$ & $\begin{array}{l}\text { Oleic } \\
\text { acid (\%) }\end{array}$ & $\begin{array}{l}\text { Linoleic } \\
\text { acid (\%) }\end{array}$ & $\begin{array}{c}\mathrm{O} / \mathrm{L} \\
\text { ratio }\end{array}$ & $\begin{array}{c}\text { Linolenic } \\
\text { acid }(\%)\end{array}$ & $\begin{array}{l}\text { Palmitoleic } \\
\text { acid (\%) }\end{array}$ & $\begin{array}{c}\text { Lauric } \\
\text { acid }(\%)\end{array}$ & $\begin{array}{l}\text { Myristic } \\
\text { acid (\%) }\end{array}$ & $\begin{array}{l}\text { Palmitic } \\
\text { acid (\%) }\end{array}$ & $\begin{array}{c}\text { Stearic } \\
\text { acid }(\%)\end{array}$ & $\begin{array}{l}\text { Arachidic } \\
\text { acid(\%) }\end{array}$ \\
\hline Dacca-1×Binachinabadam-4 & $-0.91^{*}$ & $4.24^{* *}$ & -2.15 & 0.40 & $5.70^{* *}$ & $-4.55^{* *}$ & -0.39 & $-0.73^{* *}$ & $-1.99 * *$ & 0.20 & $-7.05^{* * *}$ \\
\hline $\begin{array}{l}\text { GC }(24)-1-1-1 \\
\times \text { Binachinabadam-4 }\end{array}$ & -0.07 & -2.65 & 0.39 & -0.09 & 2.63 & -0.31 & 0.20 & 0.23 & -1.23 & 0.11 & $3.63^{*}$ \\
\hline $\begin{array}{l}\text { China Badam } \\
\times \text { Binachinabadam }-4\end{array}$ & $1.14^{* *}$ & -2.56 & 2.77 & -0.25 & 0.56 & 0.53 & -0.22 & -0.23 & $-2.20^{* *}$ & 0.65 & $7.33^{* *}$ \\
\hline GC (24)-1-1-1×Dacca-1 & -0.43 & -0.25 & 2.52 & -0.37 & $-3.03^{* *}$ & 0.08 & 0.29 & -0.01 & 0.51 & -0.23 & -2.92 \\
\hline China Badam $\times$ Dacca -1 & 0.23 & -1.88 & 0.94 & -0.43 & 1.46 & 0.72 & -0.23 & 0.06 & -0.21 & $-2.20^{* *}$ & $-14.42^{* *}$ \\
\hline China Badam $\times$ GC (24)-1-1-1 & 0.12 & $-8.01^{* *}$ & $7.55^{* *}$ & $2.08^{* *}$ & -2.72 & 1.26 & -0.12 & -0.15 & 0.71 & -0.25 & -0.28 \\
\hline SE (rij) & 0.22 & 0.78 & 1.08 & 0.25 & 0.92 & 0.53 & 0.18 & 0.19 & 0.55 & 0.31 & 0.62 \\
\hline
\end{tabular}

Note: * and ${ }^{* *}$ represent indicates significant at $5 \%$ and $1 \%$ level of probability, respectively

Table 8. Percentage of heterosis for oil content and fatty acid compositions obtained from a $4 \times 4$ diallel crossing experiment of groundnut

\begin{tabular}{|c|c|c|c|c|c|c|c|c|c|c|c|}
\hline Character $x$ Crosses & $\begin{array}{l}\text { Oil } \\
\text { (\%) }\end{array}$ & $\begin{array}{l}\text { Oleic } \\
\text { acid }\end{array}$ & $\begin{array}{l}\text { Linoleic } \\
\text { acid }\end{array}$ & $\begin{array}{c}\mathrm{O} / \mathrm{L} \\
\text { ratio }\end{array}$ & $\begin{array}{l}\text { Linolenic } \\
\text { acid }\end{array}$ & $\begin{array}{l}\text { Palmitoleic } \\
\text { acid }\end{array}$ & $\begin{array}{l}\text { Lauric } \\
\text { acid }\end{array}$ & $\begin{array}{l}\text { Myristic } \\
\text { acid }\end{array}$ & $\begin{array}{l}\text { Palmitic } \\
\text { acid }\end{array}$ & $\begin{array}{l}\text { Stearic } \\
\text { acid }\end{array}$ & $\begin{array}{l}\text { Arachidic } \\
\text { acid }\end{array}$ \\
\hline Binachinabadam- $4 \times$ Dacca- 1 & -1.42 & 0.96 & $-55.97 *$ & 2.52 & $468.43^{* *}$ & $-49.57^{*}$ & $-54.91 *$ & -8.53 & $-46.36^{*}$ & 23.79 & 8.76 \\
\hline $\begin{array}{l}\text { Binachinabadam-4 } \\
\times \text { GC (24)-1-1-1 }\end{array}$ & $7.65^{* *}$ & $35.89 *$ & 6.31 & 0.15 & $171.04^{* *}$ & 28.54 & $323.61^{* *}$ & $65.93^{*}$ & $35.63^{*}$ & -13.27 & $46.43^{* *}$ \\
\hline $\begin{array}{l}\text { Binachinabadam-4 } \\
\times \text { China Badam }\end{array}$ & $15.04^{* *}$ & -20.09 & -25.59 & 0.05 & $152.17^{* *}$ & 2.16 & $-73.07^{*}$ & $-67.77^{*}$ & -17.31 & 0.15 & $65.87^{* *}$ \\
\hline Dacca-1 $\times$ Binachinabadam-4 & 3.89 & $-29.13^{*}$ & $-42.83^{*}$ & 0.21 & $293.23^{* *}$ & $81.59^{* *}$ & $-43.46^{*}$ & $29.57^{*}$ & -13.35 & 7.78 & -9.26 \\
\hline Dacca-1 × GC (24)-1-1-1 & $-7.58^{* *}$ & 8.59 & -29.25 & 1.95 & $77.96^{*}$ & 29.86 & $-88.11^{* *}$ & $-94.57^{* *}$ & $-36.95^{*}$ & $107.6^{* *}$ & 11.54 \\
\hline Dacca-1 $\times$ China Badam & $7.99 * *$ & -2.87 & $-57.09 *$ & $4.13^{*}$ & $186.08^{* *}$ & $44.63^{*}$ & $-97.31^{*}$ & $-93.22^{* *}$ & $-52.27 *$ & -39.64 & $86.46^{* *}$ \\
\hline $\begin{array}{l}\text { GC (24)-1-1-1 } \times \\
\text { Binachinabadam-4 }\end{array}$ & $7.92^{* *}$ & $61.31^{* *}$ & 10.05 & 0.41 & $117.14^{*}$ & $40.00^{*}$ & $170.39 * *$ & -0.63 & $74.20^{* *}$ & -19.91 & -23.65 \\
\hline GC (24)-1-1-1 × Dacca-1 & $-5.48^{*}$ & 10.60 & $-47.55^{*}$ & 1.96 & $108.44^{* *}$ & 27.41 & $-97.07^{* *}$ & $-93.99 * *$ & $-47.13^{*}$ & $104.42^{* *}$ & 11.54 \\
\hline GC (24)-1-1-1 × China Badam & $4.14^{*}$ & $-51.71 * *$ & -8.40 & 0.72 & $61.30^{*}$ & 17.17 & $-91.75^{*}$ & $-54.07^{*}$ & -31.57 & $275.88^{* *}$ & 9.56 \\
\hline $\begin{array}{l}\text { China Badam } \\
\times \text { Binachinabadam }-4\end{array}$ & $11.00^{* *}$ & -5.11 & $-44.45^{*}$ & 1.15 & $142.38^{* *}$ & -12.17 & $-57.91^{*}$ & $-50.84^{*}$ & 26.39 & $-38.90 *$ & $-56.45^{* *}$ \\
\hline China Badam $\times$ Dacca-1 & $8.48^{* *}$ & 10.70 & $-69.96^{* *}$ & $10.48^{* *}$ & $168.55^{* *}$ & 19.94 & $-91.97^{*}$ & $-95.72 *$ & $-48.38 *$ & $387.30^{* *}$ & $35.65^{*}$ \\
\hline China Badam $\times$ GC (24)-1-1-1 & $4.14^{*}$ & 0.22 & $-70.68 * *$ & $25.64^{* *}$ & $83.25^{*}$ & -18.63 & $-82.73^{*}$ & $-41.19 *$ & $-49.05^{*}$ & $299.48^{* *}$ & $134.34^{* *}$ \\
\hline
\end{tabular}

* and ${ }^{* *}$ indicates significant at $5 \%$ and $1 \%$ level of probability, respectively

hybrids for oil content as well as fatty acid contents have been reported when the introduced germplasm were crossed in diallel mating scheme $(19,20)$. In several other studies, highly significant differences for fatty acid profiles (oleic, linoleic, $\mathrm{O} / \mathrm{L}$ ratio, linolenic and palmitoleic, lauric, myristic, stearic, stearic, palmitic, arachidic acid) have also been reported (19, 21, 22).

\section{Oil content in the parents and hybrids}

The oil content values of our studied varieties were ranged from 49.00 to $51.73 \%$ whereas among the hybrids and reciprocal hybrids the values ranged from $46.93-55.40 \%$ (Table 3). According to the published reports, the oil content values of peanut varieties in different market types were ranged from 42.0 to $53.8 \%$ in the Spanish type and $43.0-48.0 \%$ in Valencia type varieties (23-25). Therefore, out results goes well in accordance with the results of other researchers. The percent of oil content in groundnut seeds has been shown to vary with the cultivar, market type and the environmental conditions under which the seeds were produced $(25,26)$. However, the significant variation for oil content in the genotypes probably due to the genetic makeup and place of their origin $(25,27)$.

\section{Unsaturated fatty acid compositions and oil quality values}

In this study, remarkable differences were observed in the unsaturated fatty acids (oleic, linoleic, linolenic and palmitoleic acid) contents among the peanut varieties and their hybrids (Table 3). Similar to our results, significant variation in oleic and linoleic acid in groundnut were also reported by others $(19,28)$. It was reported that the ratios of oleic acid to linoleic acid $(\mathrm{O} / \mathrm{L}$ ratio) determine the quality, storability and shelf-life of groundnut and its products (29). Additionally, high oleic acid peanut has longer shelf-life than low-oleic groundnut. In the present experiment, the means $\mathrm{O} / \mathrm{L}$ ratio of peanut parents and hybrids were ranged from 0.95-4.87. Similar to our results, Gulluoglu et al. (28) were also reported a similar range of $\mathrm{O} / \mathrm{L}$ ratio however the fatty acids content in groundnut oil is affected by variety, seasonal variation, genotype, location, air and soil moisture, soil nutrient, planting date, moisture availability, growing condition and maturity $(19,28,29)$.

\section{Saturated fatty acids composition}

Palmitic and stearic acids are the major saturated fatty acids in groundnut oil however the other saturated fatty acids like lauric, myristic and 
arachidic acids are also present smaller quantities (25). In the present study, plamitic and stearic acid were ranges from 7.64-12.52\% and 0.89-5.26\%, respectively (Table 3 ). Our results are in agreement with the results of other researchers who reported that the palmitic acid content of peanut cultivars was varied between 8.6-14.1\% and stearic acid between $1.6-3.7 \%$ (24, 29-32). Recently, it was reported that palmitic and stearic acids percentage values of the peanut varieties were varied between 10.04-12.68\% and 2.32- 3.36\%, respectively (25). Significant differences for palmitic and stearic acids percentage among the cultivars are attributable to the genetic makeup and place of their origin (27). Additionally, saturated fatty acid compositions in groundnut oil are strongly influenced by genotype, growing season and harvesting time $(24,26)$.

\section{Combining ability analysis for oil content and fatty acids composition}

The variance for combining ability showed highly significant positive GCA and SCA for oil content and fatty acid compositions which indicates the presence of both additive and non-additive gene action on these traits expression. Beside this, significant RCA effects indicate the influence of maternal effect on the traits. The variances due to dominance deviation $\left(V_{D}\right)$ for oil and all fatty acids contents were much higher than the additive deviation $\left(V_{A}\right)$ suggested predominance of non-additive gene action for the inheritance of these traits (Table 4). The involvement of both additive and non-additive gene action for the trait (oil content) has been reported by others (21, 33, 34). However, few studies reported only additive gene effect in controlling the oil content $(19,22)$. Additionally, non-additive genetic effects for oil content in Brassica have also been reported by others $(35,36)$. Similar to oil traits, both additive and nonadditive gene action for unsaturated fatty acids composition were also reported by others viz., oleic acid $(21,34,36,38)$, linoleic acid $(21,34,38,39)$, linolenic acid (40). In addition, additive and nonadditive gene action for unsaturated fatty acids were also reported by others such as myrsitic acid (2); stearic acid (19, 37), palmitic acid (37, 38), and arachidic acid (37). However, only additive gene action for linoleic acid was also reported (19). Research on gene effect of palmitoleic acid in groundnut is unexpectedly rare. In sunflower, reports are on additive gene effect for palmitoleic acid inheritance (41). In contrast to our results of saturated fatty acids, only additive gene effect for saturated fatty acids were also reported such as myristic acid (22), plamitic acid $(42,43)$ and arachidic acid $(43,44)$.

\section{Heterosis for oil and fatty acid contents}

Among the cross combinations, Binachinabadam $-4 \times$ China Badam and its reciprocal cross were showed significantly higher positive mid-parent heterosis for oil content (Table 8) which indicates that both additive and non-additive gene action with significant maternal effect in controlling the trait. Similar to our results, significant positive heterosis oil content in both hybrid and reciprocal hybrids were also reported (45). Significant and non-significant negative heterosis for oil content were also found in the cross Dacca-1 $\times$ GC (24)-1-1-1and its reciprocal cross which is in accordance with the earlier results (46). In case of oleic acid, highly significant positive correction was found in the cross GC (24)-1-1-1 and Binachinabadam-4 and its reciprocals. Importantly, linoleic acid content in most of the crosses and reciprocal crosses showed significant negative heterosis. Similar findings were also reported in mustard (Brassica juncea L.) (46). The O/L ratio showed positive heterosis for all of the crosses and their reciprocals whereas the GC (24)-1-1-1× China Badam showed the highest significant positive heterosis. Heterosis for linolenic acid content showed significant positive for all of the crosses whereas the highest mid-parent heterosis was found from the cross Binachinabadam- $4 \times$ Dacca- 1 . A similar positive result for linolenic acid content was also reported by other researchers while studying the diallel cross (47) in mustard. In case of palmitic acid, both positive and negative mid-parent heterosis were found however, the highest positive heterosis was found from the cross Dacca-1 $\times$ Binachinabadam-4. Mid-parent heterosis for saturated fatty acids (lauric acid, myristic acid, stearic acid, palmitic acid and arachidic acid) showed both positive and negative values. Importantly, negative heterosis of this traits are desirable and the highest negative heterosis for lauric acid and stearic acid was found from the cross Dacca$1 \times$ China Badam, myristic acid from the cross Dacca$1 \times$ GC (24)-1-1-1, palmitic acid from the cross China Badam $\times$ GC (24)-1-1-1, and arachidic acid from the cross China Badam $\times$ Binachinabadam-4. Similar negative heterosis for saturated fatty acid was also reported in sunflower (48). Importantly, in most of the cases different cross combination exhibited higher superiority in hybrid, therefore, they can be used in isolating potential lines and to break oil decreasing and low quality barrier in groundnut. Therefore, these superior crosses are also expected to produce transgressive segregants.

\section{Conclusion}

In this study, significantly higher GCA for oil content, $\mathrm{O} / \mathrm{L}$ ratio and linolenic acid was observed in the parent China Badam whereas Binachinabadam-4 showed significantly higher GCA for oleic acid and linoleic acid. So, China Badam and Binachinabadam-4 could be selected as best general combiners for these traits. Best SCA performances were observed from the crosses Dacca- $1 \times$ China Badam and Binachinabadam- $4 \times$ China Badam for oil, oleic and linolenic acid content. They could be promising combiner for improving oil content and essential fatty acids. Significant heterosis was also observed in these cross combinations for these traits. Additionally, the cross China Badam $\times$ GC (24)-1-1-1 showed a higher $\mathrm{O} / \mathrm{L}$ ratio $(>4)$ along with lower level of saturated fatty acids content. The results of the present study also revealed that both additive and non-additive gene action contributes in controlling the oil and fatty acid traits however predominance of non-additive gene effect was observed along with maternal effects. So, selection in later generation 
would be more effective in developing new varieties for higher oil and healthy fatty acid compositions.

\section{Acknowledgements}

Authors gratefully acknowledge the financial support provided by Ministry of Agriculture, Government of the People's Republic of Bangladesh.

\section{Authors' contributions}

MAK Azad conceived the idea and designed the experiment. MR Sikder, SH Bhuiya, KS Arefin, MMH Shohag carried-out the experiment and analyzed the data. MAH and MAK Azad supervised the experimental work. MR Sikder and MAH wrote the initial manuscript. MAK Azad did the proof-reading of the final draft. All authors provided critical feedback and helped to shape of the final manuscript.

\section{Conflict of interests}

Authors do not have any conflict of interests to declare.

\section{References}

1. Sebei K, Gnouma A, Herchi W, Sakouhi F, Boukhchina S Lipids, proteins, phenolic composition, antioxidant and antibacterial activities of seeds of peanuts (Arachis hypogaea L.) cultivated in Tunisia. Biological Research. 2013;46(3);25763. https://doi.org/10.4067/S0716-97602013000300006

2. Shasidhar Y, Vishwakarma MK, Pandey MK, Janila P, Variath MT, Manohar SS, Nigam SN, Guo B, Varshney RK. Molecular mapping of oil content and fatty acids using dense genetic maps in groundnut (Arachis hypogaea L.). Frontiers in Plant Science. 2017;8:794. https://doi.org/10.3389/fpls.2017.00794

3. Zahran HA, Tawfeuk HZ. Physicochemical properties of new peanut (Arachis hypogaea L.) varieties. Oilseeds and fats Crops and Lipids. 2019;26:19. https://doi.org/10.1051/ocl/2019018

4. Variath MT, Janila P.2017. Economic and academic importance of peanut. In: The peanut genome. Cham: Springer, pp. 7-26. https://doi.org/10.1007/978-3-319-63935-2_2

5. Miah MA, Mondal MRI. Oilseeds sector of Bangladesh: challenges and opportunities. SAARC Journal of Agriculture.2017;15(1):161 https://doi.org/10.3329/sja.v15i1.33146

6. Dean LL, Davis JP, Sanders TH. Groundnut (peanut) oil. In: Vegetable Oils in Food Technology: Composition, Properties and Uses. $2^{\text {nd }}$ ed., Blackwell Publishing Ltd.2011. pp.225-42. https://doi.org/10.1002/9781444339925.ch8

7. Liao BS. The Groundnut. Wuhan: Hubei Press for Science and Technology; 2003.

8. Mozingo RW, O'keefe SF, Sanders TH. Improving shelf life and salted in shell peanut using high oleic fatty acid chemistry. Peanut $\quad$ Science. 2004;31:40-45. https://doi.org/10.3146/pnut.31.1.0009

9. Vassiliou E.K., Gonzalez A., Garcia C., et al. Oleic acid and peanut oil high in oleic acid reverse the inhibitory effect of insulin production of the inflammatory cytokine TNF-a both in vitro and in vivo system. Lipids in Health and Disease. 2009; 8:25. https://doi.org/10.1186/1476-511X-8-25

10. Barbour JA, Howe PR, Buckley JD, Bryan J. Coates AM. Effect of 12 weeks high oleic peanut consumption on cardio-metabolic risk factors and body composition. Nutrients. 2015;7:7381-98. https://doi.org/10.3390/nu7095343
11. Mercel LC, Wynne JC, Young CT. Inheritance of fatty acid content in peanut oil. Peanut Science. 1990;17:17-21. https://doi.org/10.3146/i0095-3679-17-1-7

12. Singkham N, Jogloy S, Suriharn B et al. Types of gene effects governing the inheritance of oleic and linoleic acids in peanut (Arachis hypogaea L.). African Journal of Biotechnology. 2012; 11:13147-52. https://doi.org/10.5897/AJB12.1498

13. Neya FB, Sanon E, Koita K, Zagre BMB, Sankara P. Diallel analysis of pod yield and 100 seeds weight in peanut (Arachis hypogaea L.) using Griffing and Hayman methods. Journal of Applied Biosciences. 2017;116:11619-27. https://doi.org/10.4314/jab.v116i1.10

14. Jambunathan AK, Singh AK, Gurtu S, Raghunathan K. Amino acid composition, fatty acid composition and levels of protease inhibitors in seeds of wild Arachis species. Oleagineux. 1993;48:415-19.

15. Danish M, Nizami M. Complete fatty acid analysis data of flaxseed oil using GC-FID method. Data in Brief. 2019; 23:10-45. https://doi.org/10.1016/j.dib.2019.103845

16. Sales N, Bartolome V, Caneda A, Gulles A, Morantte RIZ, Nora L, Raquel AM, Relente CE, Talay D, Ye G. PB Tools software for Plant Breeding. International Rice Research Institute, College, Losbanon, Laguna. 2013.

17. Griffing B. Concept of general and specific combining ability in relation to diallel crossing systems. Australian Journal of Biological Sciences. https://doi.org/10.1071/BI9560463

18. Rai B. Heterosis Breeding. Agro-biological Publication, Delhi, India. 1979:183.

19. Amoah AR, AkromahR, Asibuo JY et al. Mode of inheritance and combining ability of oleic acid content in groundnut (Arachis hypogaea L.). Ecological Genetics and Genomics, 2020 17:100064. https://doi.org/10.1016/j.egg.2020.100064

20. Mourad K. Diallel analysis of seed yield, its components and oil content in peanut. Journal of Plant Production. 2021;12(1):1-9. https://doi.org/10.21608/jpp.2021.53621.1009

21. Singkham N, Jogloy S, Kesmala T, Swatsitang P, Jaisil P, Puppala N, Patanothai A. Estimation of heritability by parentoffspring regression for high-oleic acid in peanut. Asian Journal of Plant Sciences. 2010;9:358-63. https://doi.org/10.3923/ajps.2010.358.363

22. Wilson NY, Baring MR, Burow MD, Rooney WL, Simpson CE. Diallel analysis of oil production components in Peanut (Arachis hypogaea L.). International Journal of Agronomy. 2013; Article ID 975701: 5 pages, 2013. https://doi.org/10.1155/2013/975701

23. Arioglu H, Bakal H, Gulluoglu L, Onat B, Kurt C. The effect of harvesting dates on some agronomic and quality characteristics of peanut (Arachis hypogaea L.) varieties grown as a main crop in Mediterranean region (Turkey). Turkish Journal of Field Crops. 2018;23(1):27-37. https://doi.org/10.17557/tjfc.414856

24. Bakal $H$, Arioglu $H$. The determination of fatty acids composition and oil quality factors of some peanut varieties having different market types at different harvesting times in main and double crop growing seasons in Mediterranean region. Turkish Journal of Field Crops 2019;24(2):221-29. https://doi.org/10.17557/tjfc.655078

25. Bakal H. Comparison of fatty acids composition and oil quality factors of different market type peanut (Arachis hypogaea L.) varieties. Authorea Preprints. 2020; https://doi.org/10.22541/au.160684332.20470764/v1

26. Isleib TG, Tillman BL, Pattee HE, Sanders TH, Hendrix KW Dean LO. Genotype by-environment interactions for seed composition traits of breeding lines in the uniform peanut performance test. Peanut Science. 2008;35(2);130-38. https://doi.org/10.3146/PS08-001.1

27. Hassan F, Manaf A, Ejaz. M. Determinants of oil and fatty acid accumulation in peanut. International Journal of Agriculture and Biology. 2005;7(6):895-99.

28. Gulluoglu L, Bakal H, Onat B, Sabagh AEL, Arioglu H Characterization of peanut (Arachis hypogaea L.) seed oil and fatty acids composition under different growing season under 
mediterranean environment. Journal of Experimental Biology and Agricultural Sciences. 2016;4(5S):564-71. https://doi.org/10.18006/2016.4(5S).564.571

29. Chaiyadee S, Jogloy S, Songsri P, Singkham N, Vorasoot N, Sawatsitang P, Holbrook C, Patanothai A. Soil moisture affects fatty acids and oil quality parameters in peanut. International Journal of Plant Production. 2013;7(1):81-96.

30. Onemli F. Impact of climate change on oil fatty acid composition of peanut (Arachis hypogaea L.) in three market classes. Chilean Journal of Agricultural Research. 2012;72(4);483. https://doi.org/10.4067/S071858392012000400004

31. Mzimbiri R, Shi AM, Liu H, Wang Q. A Review: peanut fatty acids determination using hyper spectroscopy imagine and its significance on food quality and safety. Food Science and Quality Management. 2014;28;90-97.

32. Chowdhury FN, Hossain D, Hosen M, Rahman S. Comparative study on chemical composition of five varieties of groundnut (Arachis hypogaea). World Journal of Agricultural Science. 2015;11(5);247-54.

33. Gaurav A, Kandhola SS, Pushp S. Genetic analysis of pod yield and confectionery traits in crosses involving bold seeded varieties of groundnut (Arachis hypogaea L.). Crop Improvement. 2010;37(1):61-65.

34. Gangadhara K, Nadaf HL. Genetic analysis of oleic acid and linoleic acid content in relation to oil quality in groundnut. Electronic Journal of Plant Breeding. 2018;9(1):283-94. https://doi.org/10.5958/0975-928X.2018.00033.9

35. Aher CD, Shelke LT, Chinchane VN, Borgaonkar SB, Gaikwad AR. Heterosis for yield and yield components in Indian mustard [Brassica juncea (L.) Czern \& Coss.]. International Journal of Plant Science. 2009;4:30-32.

36. Sabaghnia N, Dehghani H, Alizadeh B, Mohghaddam M. Heterosis and combining ability analysis for oil yield and its components in rapeseed. Australian Journal of Crop Science. 2010;4(6):390-97.

37. Aruna R, Nigam SN. Inheritance of fatty acid content and related quality traits in groundnut, Arachis hypogaea L. Journal of Oilseeds Research. 2009;26(1):10-17.

38. Singkham N, Jogloy S, Kesmala T et al. Combining ability for oleic acid in peanut (Arachis hypogaea L.). Sabrao Journal of Breeding and Genetics. 2011;43:59-72.

39. Desmae H, Janila P, Okori P, Pandey MK, Motagi BN, Monyo E, Mponda O, Okello D, Sako D, Echeckwu C, Oteng-Frimpong R, Miningou A, Ojiewo C, Varshney RK. Genetics, genomics and breeding of groundnut (Arachis hypogaea L.). Plant Breeding. 2019;138(4):425-44. https://doi.org/10.1111/pbr.12645

40. Patel MS Chauhan RM. Study on gene action, heterosis and inbreeding depression for yield and quality traits in castor (Ricinus communis L.). Journal of Oilseeds Research. 2013;30(1);138-43.
41. Tyagi V, Dhillon SK, Kaur G. Gene action for oil content and quality in diverse cytoplasmic sources in sunflower under varied moisture environments. Helia. 2020;43(73):151-66 https://doi.org/10.1515/helia-2020-0020

42. Barkley NA, Isleib GT, Wang ML, Pittman RN. Genotypic effect of ahFAD2 on fatty acid profiles in six segregating peanut (Arachis hypogaea L.) populations. BMC Genetics. 2013;14:62. https://doi.org/10.1186/1471-2156-14-62

43. Achola E, Tukamuhabwa P, Adriko J, Edema R, Mwale SE, Gibson P, Okello DK. Composition and variation of fatty acids among groundnut cultivars in Uganda. African Crop Science Journal. 2017;25(3);291-99. https://doi.org/10.4314/acsj.v25i3.3

44. Wang ML, Khera P, Pandey MK, Wang H, Qiao L, Feng S et al Genetic mapping of QTLs controlling fatty acids provided insights into the genetic control of fatty acid synthesis pathway in peanut (Arachis hypogaea L.). PLoS ONE. 2015;10(4):e0119454.

https://doi.org/10.1371/journal.pone.0119454

45. Boraiah KM, Goud S, Gejli K, Konda CR, Babu HP. Heterosis for yield and yield attributing traits in groundnut (Arachis hypogaea L.). Legume Research. 2012;35:119-25.

46. Ali N, Bakht J, Naveed K. Heterosis studies for some fatty acids composition of Indian Mustard (Brassica juncea L.). Journal of Animal and Plant Sciences. 2015;25:587-92.

47. Shrimali TM, Chauhan RM, Gami RA, Patel PT. Diallel analysis in Indian mustard (Brassica juncea L. Czern \& Coss.) Electronic Journal of Plant Breeding. 2016;7(4):919-24 https://doi.org/10.5958/0975-928X.2016.00125.3

48. Shamshad M, Dhillon SK, Gurpreet K. Heterosis for oil content and oil quality in sunflower (Helianthus annuus L.). Current Advances in Agricultural Sciences. 2016;8(1):44-48. https://doi.org/10.5958/2394-4471.2016.00010.1

Additional information

Peer review information: Plant Science Today thanks Sectional Editor and the other anonymous reviewers for their contribution to the peer review of this work.

Reprints and permissions information is available at https://horizonepublishing.com/journals/index.php/PST/open_access_policy

Publisher's Note: Horizon e-Publishing Group remains neutral with regard to jurisdictional claims in published maps and institutional affiliations.

To cite this article: Sikder M R, Azad M A K, Bhuiya S H, Arefin K S, Shohag M M $\mathrm{H}$, Hossain M A. Combining ability and heterosis analyses for oil and healthy fatty acid composition in groundnut (Arachis hypogaea L.). Plant Science Today. 2021;8(3):732-740. https://doi.org/10.14719/pst.2021.8.3.1239

Plant Science Today, published by Horizon e-Publishing Group, is covered by Scopus, Web of Science, BIOSIS Previews, Clarivate Analytics, etc. See https://horizonepublishing.com/journals/index.php/PST/indexing_abstracting 